\title{
Multiscale modeling with GENESIS 3, using the G-shell and Python
}

\author{
Armando L Rodriguez ${ }^{1 *}$, Hugo Cornelis ${ }^{2}$, David Beeman ${ }^{3}$, James M Bower ${ }^{1}$ \\ From Twenty First Annual Computational Neuroscience Meeting: CNS*2012 \\ Decatur, GA, USA. 21-26 July 2012
}

The CBI architecture [1] being used as the basis of GENESIS 3 (G-3) allows a single model-container to be used to describe a model spanning many levels of scale. This feature allows a user to transparently run multi-scale simulations. As will be described in further detail during the workshop "Multi-Scale Modeling in Computational Neuroscience II: Challenges and Opportunities", the CBI architecture contains a communication component to upscale and downscale numerical variables when moving across different levels of scale. These new capabilities and advances in G-3 usability also allow interfacing with many Python graphical tools (e.g. wxPython, matplotlib), potential web interfaces (e.g. Django), and other independent modules (e.g. Chemesis-3) for use in simulations that cover multiple levels of scale. Progress in developing Python interfaces to G-3 [2], combined with recent implementation of network and biochemical modeling capabilities in G-3 have allowed us to construct a new series of self-guided hands-on modeling tutorials. These are being introduced at the Introduction to Genesis 3 Workshop held in Luebeck, Germany 30 April - 5 May 2012 (https://www.gradschool.uni-luebeck.de/index.php? $\mathrm{id}=366)$.

This poster provides an introduction to these new modeling capabilities, and to the new instructional material. Additions to the existing G-3 tutorials on use of the Gshell cover network creation commands and the use of the Chemesis-3 module. The rewritten version of the tutorial "Creating large networks with GENESIS" demonstrates the use of Python scripting to create cortical network models in G-3. The tutorial "Adding a GUI to G-3 simulations" shows users how to leverage the Python programming interface to construct visual tools.

\footnotetext{
* Correspondence: dbeeman@colorado.edu

'Barshop Institute, University of Texas Health Science Center, San Antonio, TX 78229, USA

Full list of author information is available at the end of the article
}

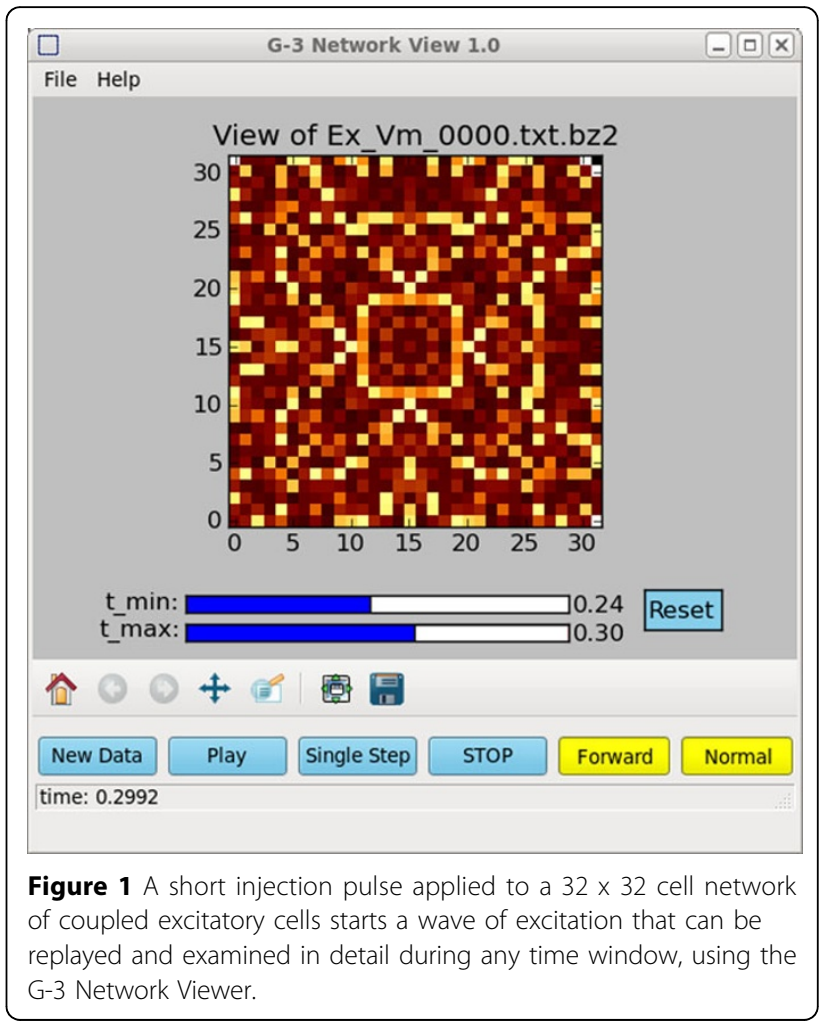

As an example, Figure 1 illustrates the use of the new G-3 Netview visualization application to display and replay an animation of the spreading excitation in the RSnet2 simulation that is the basis of the GENESIS network modeling tutorial.

\section{Acknowledgements}

Armando L. Rodriguez and David Beeman are partially supported by $\mathrm{NIH}$ grant 3 R01 NS049288-06S1 to James M. Bower.

\section{Biomed Central}




\section{Author details}

'Barshop Institute, University of Texas Health Science Center, San Antonio, TX 78229, USA. ${ }^{2}$ Department of Neurophysiology, Catholic University of Leuven, Leuven, 3000, Belgium. ${ }^{3}$ Department of Electrical, Computer, and Energy Engineering, University of Colorado, Boulder, CO 80309, USA.

Published: 16 July 2012

\section{References}

1. Cornelis H, Coop AD, Bower JM: A Federated Design for a Neurobiological Simulation Engine: The CBI Federated Software Architecture. PLOS ONE 2005, 7(1):e28956, doi:10.1371/journal.pone.0028956.

2. Cornelis H, Rodriguez AL, Coop AD, Bower JM: Python as a Federation Tool for GENESIS 3.0. PLOS ONE 2005, 7(1):e29018, doi:10.1371/journal. pone.0029018.

doi:10.1186/1471-2202-13-S1-P176

Cite this article as: Rodriguez et al: Multiscale modeling with GENESIS 3, using the G-shell and Python. BMC Neuroscience 2012 13(Suppl 1):P176. 